\title{
Effect of early and late syphilis on central nervous system: cerebrospinal fluid changes and neurological deficit
}

\author{
R V W VAN EIJK,* E Ch WOLTERS, $\dagger$ J A TUTUARIMA, $\ddagger$ E A H HISCHE, $\ddagger$ J D BOS, $\S$ L VAN \\ TROTSENBURG, $\ddagger \mathrm{G}$ A J DE KONING, AND H J van der HELM $\ddagger$ \\ From the *National Institute of Public Health and Environmental Hygiene, Laboratory for Bacteriology, \\ Bilthoven, the †Department of Neurology, Academic Hospital of the Free University, the Departments of \\ $¥ N e u r o l o g y$ and $\S D e r m a t o l o g y$, Academic Hospital of the University of Amsterdam, and the 1 Department of \\ Infectious Diseases, Municipal Health Service, Amsterdam, the Netherlands
}

SUMMARY Neurological examination and investigation of the cerebrospinal fluid (CSF) was performed on 24 patients with early and 180 patients with late syphilis.In 21 (12\%)patients with late syphilis positive CSF treponemal test results and neurological deficits suggestive of symptomatic neurosyphilis were found. Concomitantly all but three patients with neurosyphilis showed one or more of the following abnormal CSF variables: CSF concentration of albumin $x 10^{3} /$ serum concentration (albumin ratio) $\geq 7.9$; mononuclear cells $>5 \mu \mathrm{l}$; ratio of CSF to serum IgG concentrations/ ratio of CSF to serum albumin concentrations (IgG index) $\geq 0.7$ or of IgM/albumin (IgM index) $\geq$ $0: 1$; or oligoclonal CSF immunoglobulins. In $20(95 \%)$ patients with neurosyphilis evidence of the production of treponemal antibodies within the central nervous system (CNS) was shown. Ten (48\%) patients with neurosyphilis had been treated previously for late syphilis. These observations emphasise the need to screen for neurosyphilis in patients with late syphilis.

Intrathecal production of treponemal antibodies was detected in six (25\%) patients with early and $44(28 \%)$ with late syphilis who did not show any neurological deficit. Intrathecal production of treponemal antibodies indicating that the CNS was affected led us to suspect asymptomatic neurosyphilis in these patients. Seventeen (11\%) patients with late syphilis but no neurosyphilis and only one (4\%) with early syphilis showed additional abnormal CSF variables.Surprisingly, six out of 22 patients with treated early and 20 out of 68 patients with treated late syphilis showed evidence of treponemal antibody production within the CNS. We do not know whether these findings indicate that the CNS was affected because of inadequate treatment or merely reflect persistent synthesis of treponemal antibodies associated with cured infection. In one (4\%) patient with early and in 21 (13\%)with late syphilis but no neurosyphilis abnormal CSF variables in the absence of positive CSF treponemal test results were observed, which excluded syphilitic inflammation of the CNS.

\section{Introduction}

Treponema pallidum is known to invade the central nervous system (CNS) within a few weeks of the original infection.Abnormalities in the CSF thought to reflect meningeal inflammation are found in $10 \%$ of patients with primary and $30 \%$ of those with secondary syphilis.' Though early syphilitic CSF changes are usually transient, some patients will have persistent CSF abnormalities as a consequence of chronic

Address for reprints: Dr R V W van Eijk, Laboratory for Bacteriology, National-Institute of Public Health and Environmental Hygiene, P O Box 1, 3720 BA Bilthoven, the Netherlands

Accepted for publication 28 June 1986 meningitis..$^{2}$ In the absence of neurological symptoms this condition is referred to as asymptomatic neurosyphilis, and people affected are at risk of developing symptomatic neurosyphilis. ${ }^{3}$ Detecting asymptomatic neurosyphilis by examinating the CSF followed by adequate treatment can prevent its progression to symptomatic disease.

The diagnostic value of traditional CSF variables for monitoring treponemal invasion of the CNS, such as increased mononuclear cell count, raised total protein concentration, and positive non-treponemal test results, has been questioned. Normal test results have often been found in patients with symptomatic neurosyphilis. ${ }^{46}$ 
Though the $T$ pallidum haemagglutination assay (TPHA) and the fluorescent treponemal antibody (FTA) test are more sensitive than the non -treponemal tests in CSF samples from patients with symptomatic neurosyphilis,${ }^{78}$ their diagnostic value in patients with syphilis who lack other evidence of CNS disease remains uncertain. A positive CSF treponemal test result could be due either to the passive transfer of antibodies from the blood or their production within the CNS.

To establish more reliable indicators of treponemal invasion of the CNS several new variables have been proposed. These include evidence of the local production of IgG, IgM, and treponemal IgG by estimating the ratios of CSF to serum IgG concentrations/ratios of CSF to serum albumin concentrations (the IgG index $),{ }^{9}{ }^{10} \mathrm{IgM} /$ albumin (IgM index),${ }^{10}$ or treponemal to total IgG, ${ }^{11}$ or by detecting oligoclonal IgG, IgM , or treponemal IgG in the CSF .1012

The study reported here was performed to evaluate variables indicating that the CNS of patients with early or late syphilis was affected. For this purpose patients were examined for neurological signs and intrathecal treponemal antibody production in relation to other signs of an inflammatory process in the CNS.

\section{Patients and methods}

CSF AND SERUM SAMPLES

CSF and serum samples were collected from 24 patients with early (less than one year's duration) syphilis and 180 patients with late (more than one year's duration) syphilis. All patients attended clinics for sexually transmitted diseases. Treatment with penicillin had been given to 22 patients with early syphilis one year before sampling and to 78 patients with late syphilis one to 15 (mean 3.5 ) years before sampling.

The diagnosis and classification of syphilis was made on the basis of history and clinical and laboratory findings. All patients were examined by the same neurologist ( $\mathrm{EChW}$ ). Evidence of a neurological deficit was based on the results of clinical examination and their correlation with underlying anatomical and physiological bases. In addition to neurological findings and psychiatric signs, special attention was given to neuro-ophthalmic signs. Chest $x$ rays and electrocardiograms were performed to exclude cardiovascular syphilis. Duration of the disease was estimated according to the patient's history, and the results of contact tracing and clinical and laboratory investigations. Treatment consisted of benzathine benzylpenicillin (Penidural) 2.4 MIU by intramuscular injection on days 1,8 , and 15 .

Patients with CSF antibodies to human immunodeficiency virus (HIV) were excluded from the study.
TESTS FOR SYPHILIS

All CSF and serum samples were tested with the TPHA (Japan Lyophilisation Company) and the Venereal Diseases Research Laboaratory (VDRL) tests. TPHA results for CSF samples were considered to be positive at a dilution of $1: 4$ or more. Serum samples were also investigated with the fluorescent treponemal antibody absorption (FTA-ABS) test, the 19S(IgM)-FTA-ABS test, and the $T$ pallidum immobilisation (TPI) test. The FTA and the (IgM)FTA test were performed on CSF samples diluted 1:5 in phosphate buffered saline (PBS).

\section{CSF AND SERUM INVESTIGATION}

CSF cell counts were performed in triplicate in a Fuchs-Rosenthal counting chamber. Albumin and IgG concentrations in CSF and serum were measured by a turbidimetric procedure. ${ }^{13}$ IgM concentrations were measured by a fluoroimmunoassay. ${ }^{14}$

The albumin ratio (CSF albumin concentration $x$ $10^{3}$ /serum albumin concentration) was estimated as a measure of blood brain barrier function. The IgG or IgM index was calculated from the ratio of CSF to serum IgG or IgM concentrations/ratios of CSF to serum albumin concentrations. ${ }^{15}$ Oligoclonal immunoglobulins in CSF were shown by the use of cellulose acetate electrophoresis. ${ }^{16} \mathrm{CSF}$ was regarded as being abnormal if the albumin ratio was $\mathbf{7 . 9}$ or more, there were more than $5 / \mu \mathrm{l}$ mononuclear cells, the IgG index was $\mathbf{0 . 7}$ or more, the IgM index was $\mathbf{0 . 1}$ or more, or there were oligoclonal immunoglobulins.

Local production of treponemal IgG was investigated by calculating the intrathecal $T$ pallidum antibody (ITPA) index from the ratio of CSF to serum TPHA IgG reciprocal titres/mg total IgG .117 Theoretically an ITPA index of more than 1 indicates that treponemal antibody production takes place within the CNS.From the standard deviations (SD) of the assays used to measure the TPHA titre and the IgG concentration, however, we calculated a cut off value of $2 \cdot 6(1 \cdot 0+3 \mathrm{SD})$.

\section{STATISTICAL ANALYSIS}

Fisher's exact test and the $\chi^{2}$ test were used to assess differences between two independent populations. Significant differences were $p<0.05$.

\section{Results}

\section{CSF CHANGES AND NEUROLOGICAL DEFICIT}

Neurological examination and investigation of the CSF and serum were undertaken in 24 patients with early and 180 patients with late syphilis. In $21(12 \%)$ patients with late syphilis at least one positive CSF treponemal test result in combination with neurological 
TABLE I Results of cerebrospinal fluid (CSF) investigation in different groups of patients with syphilis

\begin{tabular}{|c|c|c|c|c|}
\hline \multirow[b]{2}{*}{ Diagnostic category } & \multirow[b]{2}{*}{$\begin{array}{l}\text { No of } \\
\text { patients }\end{array}$} & \multicolumn{2}{|c|}{ No (\%) with CSF treponemal test results: } & \multirow[b]{2}{*}{$\begin{array}{l}\text { Positive } \S \text { and } \\
\text { CSF variables } \\
\text { abnormal }\end{array}$} \\
\hline & & $\begin{array}{l}\text { Negative } \dagger \text { and } \\
\text { CSF variables } \\
\text { abnormal } \ddagger\end{array}$ & $\begin{array}{l}\text { Positive } \S \text { and } \\
\text { CSF variables } \\
\text { normal }\end{array}$ & \\
\hline Neurosyphilis & 11 & 0 & $2(18)$ & $9(\overline{82})$ \\
\hline Treated* neurosyphilis & 10 & 0 & $1(10)$ & $9(90)$ \\
\hline Late syphilis & 91 & $10(11)$ & 37 (41) & $19(21)$ \\
\hline Treated late syphilis & 68 & $11(16)$ & $25(37)$ & 11 (16) \\
\hline Early syphilis & 2 & 0 & 0 & $2(100)$ \\
\hline Treated early syphilis & 22 & $1(5)$ & $11(50)$ & $2(9)$ \\
\hline
\end{tabular}

* Previously treated for late syphilis.

† Treponema pallidum haemagglutination assay (TPHA) and fluorescent treponemal antibody (FTA) test.

$\ddagger$ Albumin ratio (CSF concentration $\times 10^{3} /$ serum concentration) $\geq 7 \cdot 9, \operatorname{IgG}$ index (ratio of CSF to serum concentration) $\geq 0 \cdot 7, \operatorname{IgM}$ index $\geq$ $0 \cdot 1$, oligoclonal immunoglobulins present, or mononuclear cell count $>5 \cdot 0 / \mu \mathrm{l}$.

$\S$ TPHA or FTA, or both.

deficit was found. According to the neurological features, 12 patients were classified as having meningovascular and the remaining nine patients as having parenchymatous forms of symptomatic neurosyphilis (a detailed study of the neurological symptomatology is in progress). Ten $(48 \%)$ of the patients with neurosyphilis had been treated previously with benzathine benzylpenicillin for late syphilis.

Table I summarises the results of CSF investigation in six groups of patients with treated and untreated early and late syphilis and neurosyphilis. Changes in the CSF were grouped into the following categories: negative CSF treponemal (TPHA or FTA) test results and abnormal CSF variables; positive CSF treponemal test results and normal CSF variables, or a combination of positive CSF treponemal test results and abnormal CSF variables.

All but three patients with neurosyphilis had positive CSF treponemal test results and abnormal CSF variables. The remaining three patients had only positive CSF treponemal test results.

Of the 91 patients with late syphilis and 68 with treated late syphilis, $37(41 \%)$ and $25(37 \%)$ respectively had positive CSF treponemal test results only, and $19(21 \%)$ and $11(16 \%)$ respectively had a combination of positive CSF treponemal test results and abnormal CSF variables. CSF changes in the absence of reactive CSF treponemal test results were found in $10(11 \%)$ patients with late syphilis and 11 (16\%) with treated late syphilis.

Eleven $(50 \%)$ patients with treated early syphilis gave positive CSF treponemal test results, two (9\%) also had abnormal CSF variables, and one $(5 \%)$ had abnormal CSF variables only. Both patients with early syphilis had positive CSF treponemal test results as well as abnormal CSF variables.
Subgrouping the patients into categories according to the presence or absence of a record of treatment did not show any significant differences.

\section{RESULTS OF TESTS FOR SYPHILIS IN CSF}

Table II compares the incidence of positive CSF test results for syphilis in the three diagnostic groups of patients with syphilis. TPHA results were generally positive more often than the results of other tests for syphilis.

Of the $21 \mathrm{CSF}$ samples from patients with neurosyphilis, all were reactive to the TPHA, 18 (90\%) to the FTA test, three (14\%) to the VDRL test, and only one (5\%) to the (IgM) FTA test. Smaller percentages of samples from patients with early and late syphilis were reactive. Samples from 89 (56\%) patients with late syphilis were TPHA positive, 56 (35\%)were reactive to the FTA, and only two (1\%) to the IgM-FTA test. Of the 24 patients with early syphilis, $15(63 \%)$ were reactive to the TPHA and six (25\%) to the FTA.

TABLE II Results of tests for syphilis in cerebrospinal fluid (CSF) from different groups of patients with syphilis

\begin{tabular}{lcclllll} 
& & \multicolumn{5}{l}{ No (\%) positive to: } \\
\cline { 3 - 7 } $\begin{array}{l}\text { Diagnostic } \\
\text { category }\end{array}$ & No of & patients & TPHA & FTA & IgM-FTA & VDRL \\
\hline Neurosyphilis & 21 & $21(100)$ & $18(90)^{*}$ & $1(5)^{*}$ & $3(14)$ \\
Late syphilis & 159 & 89 & $(56)$ & $56(35)$ & $2(1)$ & 0 \\
Early syphilis & 24 & 15 & $(63)$ & $6(25)$ & 0 & 0 \\
\hline
\end{tabular}

TPHA $=$ Treponema pallidum haemagglutination assay.

FTA $=$ Fluorescent treponemal antibody test.

VDRL $=$ Venereal Diseases Research Laboratory test.

* Only 20 patients tested. 
TABLE III Incidence of intrathecal Treponema pallidum antibody (ITPA) production (ITPA index $>2 \cdot 6$ ) and abnormal* cerebrospinal fluid (CSF) variables in different groups of patients with syphilis

\begin{tabular}{lcrrr}
\hline & & \multicolumn{3}{l}{$\begin{array}{l}\text { No (\%) producing ITPA } \\
\text { with CSF variables: }\end{array}$} \\
\cline { 3 - 6 } $\begin{array}{l}\text { Diagnosis } \\
\text { category }\end{array}$ & $\begin{array}{l}\text { No of } \\
\text { patients }\end{array}$ & Normal & Abnormal & Total \\
\hline Neurosyphilis & 21 & $3(14)$ & $17(81)$ & $20(95)$ \\
Late syphilis & 159 & $27(17)$ & $17(11)$ & $44(28)$ \\
Early syphilis & 24 & $5(21)$ & $1(4)$ & $6(25)$ \\
\hline
\end{tabular}

ITPA index = ratio of CSF to serum IgG reciprocal titres in the $T$ pallidum haemagglutination assay/mg total $\mathrm{IgG}$.

- Albumin ratio (CSF concentration $\times 10^{3} /$ serum concentration) $\geq$ 7.9, Ig G index (ratio of CSF to serum concentrations/ratio of CSF to serum albumin concentrations) $\geq 0.7$, IgM index $\geq 0.1$, oligoclonal immunoglobulins present, or mononuclear cell count $>5 / \mu l$.

\section{INTRATHECAL PRODUCTION OF TREPONEMAL} ANTIBODY

To establish the incidence of intrathecal treponemal antibody production in the three diagnostic groups of patients with syphilis, the ITPA index was calculated from the ratio of CSF to serum TPHA IgG reciprocal titres/mg total IgG. The results are summarised in table III.

All but one patient with neurosyphilis gave an ITPA index value greater than the cut off value of 2.6 (mean $21 \cdot 3$, range $2 \cdot 8$ to 88 ), reflecting treponemal antibody production within the CNS. One patient who had been treated previously for late syphilis had an ITPA index of 1.8. An increased ITPA index and abnormal CSF variables were found in $17(81 \%)$ patients.

Of the 159 patients wth late syphilis $44(28 \%), 20$ of them treated, had an IPTA index greater than the cut off value (mean $8 \cdot 7$, range 2.6 to 43.5 ). Seventeen (11\%) of them also had abnormal CSF variables.
Of the 24 patients with early syphilis, six (25\%), all treated, had an ITPA index value greater than the cut off value (mean $6 \cdot 0$, range $4 \cdot 1$ to $10 \cdot 2$ ). One (4\%) also had abnormal CSF variables.

INCIDENCE OF SINGLE ABNORMAL CSF VARIABLE Table IV shows the incidence of single abnormal CSF variables in different groups of patients with syphilis.

Of the 21 patients with neurosyphilis, only four (19\%) had a raised CSF mononuclear cell count, 13 (62\%) had a raised IgG index, nine (43\%) had oligoclonal CSF immunoglobulins , and six (29\%) had a raised IgM index. The blood brain barrier was damaged in eight $(38 \%)$ cases.

The presence of CSF oligoclonal immunoglobulins ( 23 cases (14\%)), a damaged blood brain barrier (18 cases $(11 \%)$ ), or a raised CSF mononuclear cell count (18 cases $(11 \%)$ ) were the variables that most often changed in late syphilis. Five patients had a raised IgM index and four (3\%) had a raised IgG index.

Of the 24 patients with early syphilis, two (8\%) had an increased IgG index or a damaged blood brain barrier, and one (4\%) had a raised mononuclear cell count or CSF oligoclonal immunoglobulins.

In comparing the percentages of single abnormal CSF variables in early and late syphilis according to the presence or absence of positive CSF treponemal test results (table IV) or to evidence of intrathecal treponemal antibody production (results not shown) no significant differences $(p>0.05)$ were observed.

\section{Discussion}

The present study showed $21(12 \%)$ patients suffering from symptomatic neurosyphilis among 180 with late syphilis. About half of those with neurosyphilis had been treated previously for late syphilis. All but one

TABLE IV Incidence of single abnormal cerebrospinal fluid (CSF) variable in different groups of patients with syphilis

\begin{tabular}{|c|c|c|c|c|c|c|c|}
\hline \multirow[b]{2}{*}{$\begin{array}{l}\text { Abnormal CSF } \\
\text { variables }\end{array}$} & \multirow{2}{*}{$\begin{array}{l}\begin{array}{l}\text { No }(\%) \text { with } \\
\text { neurosyphilis }\end{array} \\
\begin{array}{l}\text { Positive } \\
(n=21)\end{array}\end{array}$} & \multicolumn{3}{|c|}{ No (\%) with late syphilis } & \multicolumn{3}{|c|}{ No (\%) with early syphilis } \\
\hline & & $\begin{array}{l}\text { Positive } \ddagger \\
(n=92)\end{array}$ & $\begin{array}{l}\text { Negative } \\
(n=67)\end{array}$ & $\begin{array}{l}\text { Total } \\
(n=159)\end{array}$ & $\begin{array}{l}\text { Positive } \neq \\
(n=15)\end{array}$ & $\begin{array}{l}\text { Negative } \\
(n=9)\end{array}$ & $\begin{array}{l}\text { Total } \\
(n=24)\end{array}$ \\
\hline $\begin{array}{l}\text { Albumin ratio* } \geq 7.9 \\
\text { Mononuclear cells } \\
>5.0 / \mu \mathrm{l}\end{array}$ & $\begin{array}{l}8(38) \\
4(19)\end{array}$ & $\begin{array}{l}11(12) \\
12(13)\end{array}$ & $\begin{array}{l}7(11) \\
6(9)\end{array}$ & $\begin{array}{l}18(11) \\
18(11)\end{array}$ & $\begin{array}{l}2(13) \\
0\end{array}$ & $\begin{array}{l}0 \\
1(11)\end{array}$ & $\begin{array}{l}2(8) \\
1(4)\end{array}$ \\
\hline $\begin{array}{l}\text { IgG index } \dagger \geq 0.7 \\
\text { IgM index } \dagger \geq 0.1 \\
\text { Oligoclonal } \\
\text { immunoglobulins }\end{array}$ & $\begin{array}{r}13(62) \\
6(29) \\
9(43)\end{array}$ & $\begin{array}{r}3(3) \\
4(4) \\
11(12)\end{array}$ & $\begin{array}{rr}1 & (1) \\
1 & (1) \\
12 & (18)\end{array}$ & $\begin{array}{r}4(3) \\
5 \quad(3) \\
23(14)\end{array}$ & $\begin{array}{ll}2(13) \\
0 \\
1 & (7)\end{array}$ & $\begin{array}{l}0 \\
0 \\
0\end{array}$ & $\begin{array}{l}2(8) \\
0 \\
1(4)\end{array}$ \\
\hline
\end{tabular}

- CSF albumin concentration $\times 10^{3} /$ serum albumin concentration.

+ Ratio of CSF to serum immunoglobulin concentrations/ratio of CSF to serum albumin concentrations.

‡ Treponema pallidum haemagglutination assay (TPHA) or fluroescent treponemal antibody (FTA) test, or both.

$\S$ TPHA and FTA test. 
patient with neurosyphilis produced intrathecal treponemal antibodies, and all but three had abnormal CSF variables. These findings emphasise the need to screen patients with late syphilis for neurosyphilis.

It has been reported that penicillin dosage regimens commonly used to treat late syphilis do not consistently reach treponemicidal concentrations in the CSF ${ }^{18} 19$ and are insufficient to treat syphilis when the CNS is affected. ${ }^{2021}$ The results of our study confirm these findings. It could not, however, be established beyond doubt whether the patients with neurosyphilis in this study who had been treated for late syphilis represented treatment failures or reinfection.

Only one patient with positive CSF treponemal test results and clinical features of neurosyphilis showed an ITPA index below the cut off value of $2 \cdot 6$. Antibiotic treatment for late syphilis two years before examination had apparently suppressed intrathecal treponemal antibody production.

Evidence of intrathecal treponemal antibody production and local synthesis of immunoglobulin, which was indicated by an increased IgG index or the presence of oligoclonal CSF bands, was found in 14 (67\%) patients with neurosyphilis. An association between treponemal antibody production within the CNS and oligoclonal CSF immunoglobulins has been established in neurosyphilis. ${ }^{17}$ Most of these oligoclonal CSF bands have been shown by antibody immunofixation to represent treponemal antibodies. ${ }^{1012}$

Intrathecal production of IgM, which is said to reflect active neurosyphilis, ${ }^{22}$ was shown by six (29\%) patients with neurosyphilis, all of whom had raised IgG indices. In only one of these patients were treponemal IgM antibodies found in the CSF. This would imply that the observed local production of IgM, in contrast to that of $\mathrm{IgG}$, is mainly non-specific and might be the result of polyclonal $B$ cell activation. Consistent with this view is the finding that treponemal IgM antibodies were not detected by antibody immunofixation in the oligoclonal CSF band of patients with neurosyphilis with an increased IgM index..$^{10}$

In most of the patients with neurosyphilis the VDRL test of the CSF gave negative results and the CSF mononuclear cell count was normal, which indicated the insensitivity of these tests as markers for invasion of the CNS by treponemes. ${ }^{45}$ The absence of increased numbers of mononuclear cells in the CSF of patients with syphilis who produce intrathecal immunoglobulins seems to be contradictory. It could, however, be assumed that most mononuclear cells reside in the depth of the brain tissue from where immunoglobulins are secreted by plasma cells into the CSF space.

Assuming that local treponemal antibody production indicates that the CNS is affected, asymptomatic neurosyphilis was found in $44(28 \%)$ patients with late syphilis who did not show any signs of neurological impairment. These patients represent about half of those giving TPHA positive results in the CSF. In 27 (17\%)patients with late syphilis and local synthesis of treponemal antibodies we found no other indications of an inflammatory process within the CNS. As the ITPA index values of patients with late syphilis and abnormal CSF variables were similar to those of patients with normal CSF, it seems reasonable to conclude that treponemal antibodies are not the main constituent of the antibody response within the CNS in patients with abnormal CSF. Whether the observed increased IgG index or oligoclonal CSF bands, or both, are caused by unspecific activation of $B$ cells due to treponemal invasion of the CNS or represent a CNS immune response to other agents remains to be established.

In 21 (13\%) patients with late syphilis we observed abnormal CSF variables in the absence of a positive CSF treponemal test. The CSF abnormalities in these patients were evidently not caused by syphilitic inflammation of the CNS.

Six out of 22 patients with treated early syphilis and 20 out of 68 patients with treated late syphilis showed evidence of local treponemal antibody production. One of our questions was whether these findings indicated inadequate treatment or solely reflected synthesis of treponemal antibodies as a scar syndrome in cases of cured infection. To find the answer it will be important to follow up these patients over extended periods to assess the outcome of increased ITPA index values and abnormal CSF variables. A continuous tendency to normalise CSF changes, or complete recovery, would indicate adequate treatment and a cured infection. Alternatively, the effects of treatment achieving treponemicidal concentrations of penicillin in the CSF ${ }^{18}{ }^{19}$ could be investigated.

Available data suggest that progression -from adequately treated early syphilis to symptomatic neurosyphilis is unlikely. Complete resolution of abnormal CSF variables is usually observed, ${ }^{23}$ and symptomatic neurosyphlis does not normally develop. ${ }^{24}$ In contrast, serious doubt has been raised about the efficacy of penicillin treatment in cases of late syphilis when the CNS is affected..$^{20}{ }^{21}$ Evidence of local treponemal antibody in patients treated for late syphilis should therefore be handled cautiously.

\section{We thank $M J$ van Niekerk and J C Compeer for carrying} out the serology tests for syphilis.

\section{References}

1. Hahn RD, Clark ES. Asymptomatic neurosyphilis: a review of literature. American Joumal of Syphilis, Gonorrhea and Venereal Diseases 1946;30:305-16.

2. Merritt HH, Adams RD, Salomon HC. Neurosyphilis. New York: Oxford University Press, 1946. 
3. Nielsen JP. Prognostic importance of spinal fluid examination in syphilis. Acta Derm Venereol [Suppl] (Stockh) 1952; 29:223-8.

4. Hooshmand H, Escobar MR, Kopf SW. Neurosyphilis. A study of 241 patients. JAMA 1972;219:726-9.

5. Kolar OJ, Burkhart JE. Neurosyphilis. British Journal of Venereal Diseases 1977;53:221-5.

6. Hagedorn HJ. Immunologie der Neurosyphilis: Ihre Bedeutung für Diagnostik and Therapie. Stuttgart: G Thieme Verlag, 1983.

7. Jaffe HW, Larssen SA, Peters M, Jove DF, Lopez B, Schroeter AL. Tests for treponemal antibody in CSF. Arch Intern Med 1978;138:252-5.

8. Kasatiya S, Birry A. Further evaluation of the microhaemagglutination test to determine treponemal antibodies in CSF. British Journal of Venereal Diseases 1980;56:77-80.

9. Luger A, Schmidt BL, Steyrer K, Schonwald E. Diagnosis of neurosyphilis by examination of the cerebrospinal fluid. British Journal of Venereal Diseases 1981;57:232-7.

10. Strandberg Pedersen N, Kam-Hansen S, Link H, Mavia M. Specificity of immunoglobulins synthesized within the central nervous system in neurosyphilis. Acta Pathol Microbiol Immunol Scand [C] 1982;90:97-104.

11. Müller F, Moskophidis M. Estimation of the local production of antibodies to Treponema pallidum in the central nervous system of patients with neurosyphilis. British Journal of Venereal Diseases 1983;59:80-4.

12. Vartal F, Vandvik B, Michaelsen TE, Loe K, Norrby E. Neurosyphilis: intrathecal synthesis of oligoclonal antibodies to Treponema pallidum. Ann Neurol 1982;1 1:35-40.

13. Hische EAH, van Meegen MT, van der Helm HJ. More precise determination of the cerebrospinal fluid IgG index. Clin Chem 1985;31:1417.

14. Hische EAH, van der Helm HJ, Out T. Estimation of IgM in cerebrospinal fluid by fluoroimmunoassay. Clin Chim Acta 1979;97:93-5.
15. Link H, Wahren B, Norrby E. Pleocytosis and immunoglobulin changes in cerebrospinal fluid and herpes virus serology in patients with Guillain-Barré syndrome. J Clin Microbiol 1979;9:305-16.

16. van der Helm HJ, Hische EAH, van Walbeek HK. Monoclonal immunoglobulins demonstrable in cerebrospinal fluid by use of cellulose acetate electrophoresis. Clin Chem 1980;26:1917-8.

17. Prange HW, Moskophidis M, Schipper HI, Müller F. Relationship between neurological features and intrathecal synthesis of IgG antibodies to Treponema pallidum in untreated and treated human neurosyphilis. $J$ Neurol 1983;230:241-52.

18. Dunlop EMC, Al-Egaily SS, Houang ET. Penicillin levels in blood and CSF achieved by treatment of syphilis. JAMA 1979;241:2538-40.

19. Polnikorn N, Witsonpanich $R$, Vorachit $M$, Vejjajiva $S$, Vejiajiva A. Penicillin concentrations in cerebrospinal fluid after different treatment regimens for syphilis. British Journal of Venereal Diseases 1980;56:363-7.

20. Short DH, Knox JM, Glicksman J. Neurosyphilis, the search for adequate treatment. Arch Dermatol 1966;93:87-91.

21. Wilner E, Brody JA. Prognosis of general paresis after treatment. Lancet 1968;ii:1370-1.

22. Oxelius V-A, Rorsman H, Laurell A-B. Immunoglobulins of cerebrospinal fluid in syphilis. British Journal of Venereal Diseases 1969;45:121-5.

23. Löwhagen GB, Andersson M, Blomshand C, Roupe G. Central nervous system involvement in early syphilis. Part 1 . Intrathecal immunoglobulin production. Acta Derm Venereol (Stockh) 1983;63:409-17.

24. Perdrup A, Jørgensen BB, Pedersen NS. The profile of neurosyphilis in Denmark. A clinical and serological study of all patients in Denmark with neurosyphilis disclosed in the years 1971-1979 incl. by Wassermann reaction (CWRM) in the cerebrospinal fluid. Acta Dermatol Venereol [Suppl] (Stockh) 1981;96:3-14. 